Revista Colombiana de Obstetricia y Ginecología Vol. 58 No. 2 • 2007 • (151-158)

Educación Médica

\title{
EXPERIENCIA DE UNA INNOVACIÓN PEDAGÓGICA
}

\author{
A pedagogy innovation experience \\ José Luis Tarazona-Méndez, M.D.* \\ Recibido: enero 30/07 - Revisado: mayo 15/07 - Aceptado: junio 5/07
}

\section{RESUMEN}

En el presente artículo de reflexión sobre cómo implementar innovaciones en educación médica, el autor parte del análisis de la experiencia de un caso de implementación de una innovación pedagógica en una facultad de medicina. Basado en una realidad encontrada, realiza un ejercicio de diagnóstico sobre los problemas que impiden un adecuado desarrollo de la innovación y formula una propuesta metodológica original de cómo implementar innovaciones pedagógicas. Dicha propuesta incluye el cuadro de mando integral como elemento organizacional y la andragogía como base teórica en el proceso de aprendizaje individual y organizacional.

Palabras clave: innovación pedagógica, cuadro de mando integral, andragogía, educación médica.

\section{SUMMARY}

The present paper regarding innovation in medical education begins by analysing experience gained from pedagogical innovation being implemented at a medical school. A diagnostic exercise was based on the organisational reality found in the school concerning the problems encountered impeding innovation and proposing original methodology for implementing pedagogical innovation. This included the Balanced Scorecard as an organisational element

\footnotetext{
* Ginecólogo. Departamento de Ginecología, Obstetricia, Fundación Santa Fe de Bogotá. Docente de cátedra, Facultad de Medicina, Universidad Militar Nueva Granada.

Correspondencia: Avenida 9 No 117-20 Consultorio 906

Correo electrónico: jo-taraz@uniandes.edu.co
}

and andragogy as the theoretical basis for individual and organisational learning.

Key words: pedagogical innovation, BSC, andragogy, medical education.

\section{INTRODUCCIÓN}

La educación cumple con una serie de propósitos dentro de los cuales está la adquisición de conocimiento. Conocimiento que es fuente de mejoramiento personal y de calidad de vida individual, el cual repercute en sí mismo y en la sociedad de la cual participa. Las instituciones universitarias como garantes que son del conocimiento y responsables de la educación y formación, tienen un papel modulador entre el conocimiento general y la adquisición y formación de conocimiento específico y el propio de los alumnos. Así como el conocimiento cambia continuamente, las instituciones universitarias deben evolucionar, ser críticas al exterior, autocríticas y capaces de modificarse, cuando condiciones externas e internas sugieran la necesidad de innovar.

El mundo médico vive en continuo cambio, que obliga a la innovación constante en la forma de entender el proceso de formación y aprendizaje; razón por la cual en una facultad de medicina se inició un proceso tendiente al estudio del Aprendizaje Basado en Problemas (ABP - Wood, D; 2003, ${ }^{1}$ Tarazona; $\left.2005,^{2}\right)$ y su aplicación como estrategia de aprendizaje, entendiendo ABP como una innovación pedagógica. 


\section{Presentación del caso}

En una facultad de medicina de la ciudad de Bogotá en el año 2003 la Decanatura, junto al comité de reforma curricular y la oficina de educación de pregrado, decidió explorar la aplicación de ABP como innovación pedagógica. Como estrategia para su implementación se decidió primero: formar docentes tutores, para lo cual se organizó un diplomado en educación médica con énfasis en ABP y medicina basada en evidencia.

En una segunda fase se propuso la implementación en pregrado en el departamento de ginecología, como piloto en la facultad. Para validar la experiencia, se planteó un estudio piloto, tipo cohorte, comparando ABP con el método tradicional con el cual no estuvieron de acuerdo los estudiantes por el temor que les generó la exposición a una nueva metodología. Sin embargo se pudo iniciar la aproximación a ella con estudiantes voluntarios.

Para el siguiente semestre académico se planteó nuevamente el estudio y esta vez no se pudo llevar a cabo porque los estudiantes del grupo que recibió ABP informaron a sus pares de su experiencia positiva y todos los estudiantes solicitaron tener una experiencia con la nueva metodología, por lo cual la jefatura del departamento decidió implementar una mezcla de ABP y clases magistrales.

La experiencia en ese semestre mostró una serie de dificultades, como incumplimiento de horarios, falta de coordinación, exceso de trabajo para los estudiantes, exceso de turnos, bases de datos insuficientes, elementos que, al parecer del autor, son producto de concepciones y algunas formas de cultura institucional, las cuales generaron de forma involuntaria alguna resistencia al cambio dentro de la organización.

Basado en la experiencia se puede entender que la innovación docente y el soporte organizacional van unidos en un todo, y que, si el entorno institucional no es el adecuado, se puede llevar al fracaso una innovación pedagógica, que en sí misma puede ser una buena alternativa. Así las cosas, de la vivencia y de la revisión teórica nace una propuesta metodológica de cómo iniciar e implantar una innovación pedagógica.

\section{Propuesta metodológica}

En la sociedad del conocimiento, este, según Duderstand (1997), ${ }^{3}$ es fuente de bienestar y mejoramiento de la calidad de vida. Tradicionalmente la universidad como institución se ha erigido como guardián del conocimiento de los diferentes saberes científicos que ha desarrollado el hombre y, a su vez, ha sido la encargada de transmitirlo a las siguientes generaciones. Esta misión la han cumplido las instituciones universitarias, las cuales desarrollaron a través del tiempo estructuras rígidas; estructuras cuestionadas y sometidas a cambios hoy, presionadas por el desarrollo de las facilidades tecnológicas que han permitido una rápida difusión del conocimiento, colectiva e individualmente, el cual se ha hecho global, en un proceso similar al de la globalización económica.

Este fenómeno ha permitido el desarrollo de redes de conocimiento informales pero igualmente operantes que la universidad en la difusión del conocimiento. Además organizaciones, científicas, académicas, cuerpos colegiados, etc., son hoy factores determinantes en el desarrollo y distribución del conocimiento. Así la universidad debe cambiar, debe considerar volverse más flexible en su operar y prepararse para los retos continuos y desarrollar su capacidad de innovación constante.

Aquí quiero recalcar cómo el uso de los ordenadores ha sido un factor fundamental en el cambio universitario, y alinearnos con los conceptos de Mason (1998), ${ }^{4}$ quien afirma que el uso de estos dispositivos transformó el quehacer educativo, hasta hacer desaparecer la diferencia conceptual entre la educación presencial y a distancia; cambió los roles tradicionales de profesor, tutor, staff de apoyo, y finalmente uno de los puntos que, para el autor es tal vez el más importante, proporciona la oportunidad, que nunca antes existió, de crear una red de estudiantes, un "espacio" de pensamiento 
colectivo, que acerca a los pares para la socialización y el intercambio ocasional, fenómeno visto en los últimos años.

Cuando se piensa en mejorar o innovar en educación siempre llega a la mente de planificadores y legos la idea de formación docente, mejoramiento docente, cambios curriculares, aspectos estos de suma importancia pero cortos en el concepto de un cambio o innovación en educación, ya que de solo llevarse esto a cabo se corre el peligro de implementar aprendizajes organizacionales parciales que no impulsan un verdadero cambio en la estructura general.

Innovar no es desechar lo existente ni cambiar por cambiar. La innovación es un proceso que debe responder a unos objetivos claros y definidos, y es según lo define Salinas (2004), ${ }^{5}$ una forma creativa de selección y organización de recursos humanos y materiales, que de una manera propia y nueva permita el logro de objetivos previamente establecidos. La innovación en su esencia implica un cambio que se logra a través de un proceso, proceso en el cual según Toffler (1985), ${ }^{6}$ se requiere de tres elementos a saber: una presión externa importante, uno o unos individuos inconformes con el orden existente y una alternativa coherente presentada en un plan o modelo consecuente con la visión de la organización.

Como todo proceso requiere de un orden y estar enmarcado dentro de presupuestos, los cuales, según Fullan (1994), ${ }^{7}$ implican el cambio de representaciones individuales, colectivas y de prácticas, el cual no resulta ni espontánea ni en forma casual, sino es deliberado e impulsado voluntariamente, comprometiendo la acción consciente y pensada de los sujetos involucrados, tanto en su gestación como en su implementación.

Ninguna estrategia arriba-abajo, abajo-arriba funciona por sí sola, sino que requiere una combinación adecuada de las mismas.

Con estas características del proceso en mente, es más fácil imaginar el orden por él requerido, así la fase inicial de movilización son todas las acciones de estudio previo, verse y evaluarse la organización, que inicia con el análisis consciente de todos los participantes de la realidad externa, realidad que se convierte en esa presión externa de que habla Toffler, continúa la movilización reflexiva internamente, reflexión que debe lograr despertar inconformidad, entendiendo esta como la necesidad de dar una respuesta activa a la presión del entorno para que genere un ambiente de discusión y estudio que pueda gestar en forma negociada y colectiva una propuesta organizada con espíritu de cuerpo. Y finalmente la implementación de acciones tendientes a solucionar las inquietudes, en un proceso continuo que debe reiniciar constantemente su ciclo.

Así las cosas se propone un modelo que sirve para la planeación e implementación de una innovación pedagógica, modelo que incluye una fase de movilización, una fase de desarrollo en la cual se propone el uso de una herramienta metodológica que permita la implementación y el seguimiento, todo lo anterior enmarcado dentro de una estrategia de aprendizaje, basada en los principios del aprendizaje adulto "andragogía”, para lograr que el aprendizaje individual se convierta en organizacional y así producir un cambio dentro del organismo universitario.

En la fase de movilización, se proponen los siguientes elementos:

Análisis del entorno. Es el análisis de la sociedad a la cual sirve la universidad, sus valores y tradiciones, conceptos y necesidades, realidades y retos que se plantean.

Análisis interno. Parte de conocer y definir su propia especificidad, proceso que se puede entender como un análisis de la cultura organizacional, la cual, según Sarasola (2004), ${ }^{8}$ son las formas de ser y de hacer como las organizaciones, en tanto que son grupos de personas, van construyendo, a lo largo de su historia en su proceso de adaptación e integración, patrones que conforman el inconsciente colectivo y que se hacen cada vez más estables. El análisis de la cultura y la definición de ese inconsciente colectivo se hace a través del estudio de las normas, mitos, símbolos, ritos, lenguaje, producciones, valores y supuestos básicos (Martínez, 2003). ${ }^{9}$ 
Análisis de la misión. Se define esta como el propósito o la razón de ser de la organización.

Análisis de la visión. Se entiende como las declaraciones que señalan el rumbo, dan la dirección y unen la organización con el futuro. Finalmente se definen los objetivos, los cuales son metas claras que permiten alcanzar la visión.

Luego de conocer la realidad, definir el porqué y para qué se desea innovar o cambiar, es importante articular las acciones dentro de un propuesta metodológica administrativa que en este caso es el cuadro de mando integral (CMI).

"Un cuadro de mando integral es un sistema de objetivos, medidas, metas e iniciativas relacionadas que colectivamente describen la estrategia de una organización y cómo esta estrategia puede ser lograda" (Narváez, 2003). ${ }^{10}$

El cuadro de mando integral como herramienta estratégica de administración fue descrito por Kaplan y Norton $(1997)^{11}$ a principios de los 90, quienes observaron la realidad de unos entornos cada vez más cambiantes, una competencia mayor por las oportunidades de participación en los mercados y la necesidad de tener herramientas mejores que el solo análisis financiero, el cual únicamente les permitía ver el pasado pero no conectarse con el futuro; desarrollaron nuevas estrategias de análisis y fue así como apareció el CMI, el cual no reemplaza al análisis financiero, sino por el contrario lo optimiza y complementa con otros puntos de vista como son: los de accionistas, de clientes, de los procesos internos, de la formación y crecimiento de la organización, visiones que le subsanan las falencias a la mirada meramente económica y que ayudan en la proyección futura de la organización.

En la construcción de un CMI se parte de una serie de preguntas relacionadas con cada una de las ópticas así:

Para los accionistas. ¿Cómo debería ser la organización para obtener el mayor rendimiento?

Para la visión del cliente se pregunta ¿cómo debería ser la organización para alcanzar la visión de la organización?
Para valorar los procesos se cuestiona ¿en qué procesos la organización debe ser excelente para satisfacer a sus accionistas y clientes?

Y para ver formación y crecimiento, ¿cómo puede la organización mantener su capacidad de cambiar y mejorar, para alcanzar su visión?

A partir de las respuestas se puede llegar a la definición de estrategias, mediante las cuales la organización crea valor para sus clientes presentes y futuros, potencia sus procesos internos para generar ventajas competitivas y asegura su recurso humano, físico y tecnológico necesario para el cumplimiento de sus metas.

Con un CMI se pretende desarrollar objetivos e indicadores derivados de la visión y la estrategia organizacional; adicionalmente se crea una estructura compacta que permite la comunicación de estrategias, crea la cultura de la discusión causaefecto entre los diferentes factores en que se basa la actividad diaria y abre un espacio sistemático para llevar a cabo esta discusión, lo cual, traducido a la acción, significa que se actúa sobre diferentes partes de la organización de la siguiente manera:

Sobre visión y estrategia: clarifica visión y estrategia.

Sobre comunicación: comunica, educa sobre objetivos, vincula las recompensas con el cumplimiento de los indicadores de acción.

Sobre planeación: establece objetivos, metas, y asigna recursos.

Sobre retroalimentación: articula la visión compartida, facilita la formación y revisión de estrategia. (Narváez, 2004). ${ }^{10}$

A pesar de las bondades teóricas y reales descritas en la literatura y la experiencia de esta metodología, tiene una serie de condicionantes que están relacionados con la capacidad de autocrítica y adaptación de las organizaciones, condiciones que de no existir se convierten en limitantes. Algunas se describen así:

- Necesidad de voluntad política clara y explícita por parte de la dirección para liderar el proceso. 
- Capacidad para verse y redefinir la organización.

- Capacidad de la alta dirección para modificar sus comportamientos.

- Capacidad de adaptar gestión a los instrumentos de medición.

- Capacidad de adaptar el presupuesto.

- Programas de formación de recurso humano.

- Exhaustivo plan de comunicación.

El CMI como herramienta, según Rezzonico, ${ }^{12}$ hace que los planificadores limiten la cantidad de indicadores estratégicos a unos clave, que reflejen la complejidad del sistema evaluado, lo que ayuda a enfocarse en las cuatro perspectivas, además del modo en que tales medidas pueden predecir el éxito en el desempeño futuro de la organización.

Existen dos aspectos que deben tenerse en cuenta adicionalmente a las condiciones planteadas previamente y son: el primero la preparación exhaustiva en los operadores de las entradas y en el procesamiento de la información para que esta sea pertinente y confiable. Y la segunda que es deseable la existencia de un sistema de red informática local para el tránsito de la información por los distintos niveles de decisión.

Por otra parte, las limitaciones para instalar y/o poner en marcha un cuadro de mando integral en la evaluación de las instituciones de educación superior dependen de una serie de factores, entre los que se destacan:

- "La posición organizacional de la persona o funcionario encargado de la implementación, la influencia real que tenga sobre la dirección.

- Las limitaciones operativas para el involucramiento del resto de la organización en la medición sistemática de los indicadores.

- Baja cualificación técnico-profesional en los responsables de implementar y en aquellos responsables de reelaborar e interpretar la información brindada.

- Intereses de los responsables de la implementación y sus superiores, incluso personales, que puedan atentar contra la libre disponibilidad de la información.
- La fragmentación observada -en general- del sistema universitario que tiende a funcionar como compartimientos estancos que desatienden su participación articulada en el sistema, generando relaciones de competencia no siempre beneficiosas para el conjunto".

A mayor dimensión de la universidad, deberá tenderse a implementarlo primero en subsistemas organizacionales (facultades, escuelas, etc.) de manera que se rompa la resistencia al cambio y ganar en aprendizajes de implementación.

El CMI no es en sí mismo un objetivo, sino un medio que pretende establecer un sistema de señales que indiquen la variación en las magnitudes verdaderamente importantes que se buscan mantener bajo vigilancia, ayudando a detectar las deficiencias en los procesos que se están llevando a cabo.

Coopera, además, en la toma de decisiones basada en información cuanticualitativamente adecuada, debiendo, la organización, orientar todo este proceso evaluatorio hacia la acción y la mejora continua y no utilizarlo bajo la extendida lógica de los premios y castigos, que terminan en tendencias al ocultamiento de informaciones negativas y errores, lo que permite que estos persistan.

Hasta este momento en el proceso ya se ha desarrollado una ruta crítica, se han definido objetivos, propósitos, metas, los cuales llevan a la implementación de las acciones; acciones de cambio que, para Fullan (1994), ${ }^{7}$ implican el desarrollo por parte de la organización y los docentes, de nuevas destrezas, comportamientos y prácticas asociadas al cambio, así como la adquisición de nuevas creencias y concepciones vinculadas al mismo.

Todo parece indicar que si seguimos una ruta nos aproximamos al éxito, sin embargo, la realidad en muchas ocasiones es diferente, $y$, a pesar de las buenas intenciones y oficios de diferentes actores dentro de la institución, los cambios no se producen. Aquí vale la pena pensar en las experiencias vividas y preguntarse sobre alguna que resultara frustrante, y surge la pregunta ¿por qué no funcionó? A lo cual se puede responder de una manera muy sencilla, se 
debe a problemas en el proceso de aprendizaje. En este momento es preciso analizar cómo el proceso de aprendizaje, necesario para el desarrollo de nuevas destrezas y creencias se da en dos niveles: uno a nivel individual y otro a nivel colectivo u organizacional.

El autor considera que en el aprendizaje individual, de acuerdo con Bachellar (1948), ${ }^{13}$ existen factores como la experiencia básica, sola y enmarcada dentro de la experiencia general, el conocimiento general en contraposición al conocimiento científico, y las barreras del lenguaje que impiden el aprendizaje y el cambio, ya que la inmovilidad genera en el sujeto una sensación de comodidad, el cambio le produce temor y de esta manera prefiere decir, así soy, así me formaron y así he sido eficiente.

Visto de otra forma, el individuo, según Senge en Kim (1993), ${ }^{14}$ en su aprendizaje, desarrolla modelos mentales, los cuales son su imagen profunda de cómo funciona el mundo, imagen que tiene el poder de influir en su acción porque le da una representación visual de su realidad. Estos modelos son más que un punto de vista, son su contexto de interacción y determinan qué material es relevantemente digno de almacenar y cuál es susceptible de desechar. Visto así, el aprendizaje es un cambio en los modelos mentales, el cual se puede dar más eficientemente a través de un programa educativo basado en los supuestos de la andragogía, los cuales se tratarán posteriormente.

A nivel organizacional Kim (1993) ${ }^{14}$ presenta diferentes formas de aprender, las cuales se basan en que a partir del aprendizaje individual y la creación de modelos compartidos se genera el aprendizaje organizacional. La descripción detallada de los modelos teóricos de ciclo sencillo, ciclo doble, etc., se escapa de este escrito, sin embargo es pertinente mencionar algunas formas de aprendizaje incompleto que tienen relevancia para el tema del presente artículo.

El aprendizaje situacional, descrito como la forma puntual como alguien en la organización soluciona un problema, solución que no se codifica y hace que la organización pierda ese aprendizaje; el aprendizaje fragmentado, que se produce cuando un individuo es muy experto en un tema determinado y su conocimiento no es utilizado por la organización, fenómeno que se presenta cuando se reúnen los más expertos en muchas áreas, pero su saber no se pone al servicio de la organización, y el aprendizaje oportunista, entre otros, son muy frecuentes en el ámbito universitario.

Sin desconocer la importancia de la caracterización de los ciclos de aprendizaje y de los tipos de aprendizaje incompletos, es más importante estudiar y encontrar dónde están los obstáculos que a nivel individual "modelos mentales" y de cultura institucional puedan presentar para el aprendizaje y la posterior implementación de la innovación.

$\mathrm{Al}$ entender que el proceso de innovación está soportado por una serie de procesos de aprendizaje, considero de la mayor relevancia proponer una práctica que sea consistente con la forma de aprender de los adultos como es la andragogía.

¿Por qué plantear andragogía? Simplemente porque en este entorno se trabaja con adultos; adultos con experiencia, conocimiento, elementos desde los cuales se inicia el aprendizaje que genera una competencia, entendiendo esta como una forma de pensar que se manifiesta en un actuar frente a una situación; de lo contrario es probable que al utilizar pedagogía de tipo tradicional no se genere el cambio requerido en los esquemas mentales, pues el sujeto de aprendizaje no siente el tema útil, aplicable y necesario en su vida, circunstancias necesarias y determinantes en el aprendizaje de los adultos.

Andragogía, según Malcom Knowles (1984), ${ }^{15}$ es el arte y la ciencia de ayudar a los adultos a aprender, "es una teoría para justificar el hecho de tratar a los adultos".

La andragogía parte de una serie de supuestos tales como que los adultos para aprender necesitan saber por qué y para qué aprender algo, antes de iniciar el proceso de aprendizaje; desean ser responsables de sus propias decisiones y ser tratados como sujetos capaces de autodireccionarse; sujetos que tienen una variedad de experiencias que repre- 
sentan una fuente de aprendizaje, la cual a su vez puede generar el sesgo de presuponer.

Visto de otra forma, Knowles (1984) ${ }^{15}$ considera que en su disposición para el aprendizaje los adultos están preparados para aprender las cosas que tengan utilidad práctica en su vida, así:

1. Los adultos deben ser parte activa de la planeación y evaluación de su instrucción.

2. La experiencia provee las bases para las actividades de aprendizaje.

3. Los adultos se interesan por aprender cosas que tienen aplicación inmediata en su vida y trabajo.

4. Los adultos aprenden mejor basados en problemas que en materias.

\section{Roles y componentes en andragogía}

Un modelo andragógico debe contar con los siguientes elementos:

1. El participante adulto

Es el principal recurso en el proceso de aprendizaje, el cual se apoya en sus propios conocimientos y experiencias pasadas, desde las cuales explora y descubre sus potenciales, talentos y capacidades. Por ello todo aprendizaje sólo puede efectuarse si existe continuidad y total congruencia, en el nivel del SER como del HACER.

2. El andragogo

Es una persona reconocida como competente en la transferencia del conocimiento, o transferencia de la experiencia, o ambas. "Persona referencia" y/o "persona experta", el andragogo desempeña varios roles: facilitador, agente de cambio, agente de relación, tutor, consultor. El andragogo facilita las interacciones interpersonales y organiza la actividad educativa, cuyo principal actor, como lo señalamos, es el participante.

3. El grupo

Para la construcción del conocimiento, la negociación y el contexto cultural son elementos fundamentales. De ahí que la construcción grupal tenga un mayor poder que la individual, y por eso el trabajo en equipos de aprendedores es el ideal.

\section{El entorno}

Se describen tres tipos de entornos:

a) El medio ambiente inmediato creado para realizar el aprendizaje, es decir, la actividad educativa en sí misma.

b) El organismo educativo que facilita los recursos y los aportes tanto profesionales como materiales.

c) Las agrupaciones sociales. Un ambiente socioemotivo adecuado para hacer propicio el proceso de aprendizaje. Así mismo, los espacios físicos y los recursos tecnológicos constituyen entre sí factores importantes para facilitar el aprendizaje.

Teniendo en cuenta los elementos descritos en la andragogía es factible diseñar los ambientes adecuados para que se pueda producir un aprendizaje de todos los implicados, se desarrollen competencias y se implemente una innovación pedagógica.

\section{CONCLUSIONES}

Con los anteriores elementos de juicio el autor concluye que para la implementación de una innovación pedagógica en el ámbito universitario son importantes, complementarios e inseparables, el marco administrativo organizacional y la apropiación del conocimiento en los niveles individual y colectivo, lo cual se puede lograr dentro de un plan estratégico, que incluya un cuadro de mando integral, soportado por una metodología educativa que lleve el conocimiento a la comunidad que aprende, en este caso todos los implicados, directivos, docentes, administradores, etc.

La estrategia de aprendizaje debe ser consistente con los principios de la andragogía, los cuales pueden ser aplicados en modelos como el aprendizaje basado en problemas, resoluciones de casos, etc. Esta forma de aprender permite una difusión y apropiación adecuada del conocimiento por todos los miembros de la comunidad, lo cual produce un cambio en los niveles individual y colectivo, que lleva a la apropiación de la innovación por la organización. 


\section{AGRADECIMIENTOS}

Agradecimientos al decano, docentes y alumnos de la Facultad de Medicina de la Universidad Militar Nueva Granada.

\section{REFERENCIAS}

1. Wood D. Abc of learning and teaching in medicine. Problem based medicine. BMJ 2003;326:328-30.

2. Tarazona JL. Reflexiones acerca del aprendizaje basado en problemas. Una alternativa en la educación médica. Rev Colomb Obstet Ginecol 2005;56:147-54.

3. Duderstand J. The future of University in an age of knowledge. Journal of Asynchronous Learning Networks 1997;1:78-87.

4. Mason R. Model of on line courses. Visitado 2007 May 15. Disponible en: http://www.sloan-c.org/ publications/magazine/v2n2/mason.asp

5. Salinas J. Innovación docente y uso de las TIC en la enseñanza universitaria. Revista Universidad y Sociedad del Conocimiento 2004;1:1-15.

6. Toffler A. The adaptative corporation. New York: McGrawHill; 1985.

7. Fullan M. Coordinating top-down and bottonup strategies for educational reform. En: Anson RJ (Comp.). Systemic reform: perspectives on personalizing education. Washington D.C.: US Department of Education Report; 1994.

8. Sarasola MR. Una aproximación al estudio de la cultura organizacional en centros educativos. Archivos Analíticos de Política Educativa 2004;12(57).

9. Martínez-Otero V. Cultura escolar y mejora de la educación. Madrid: Universidad Complutense; 2003.

10. Narváez C. Propuesta para la implementación de un cuadro de mando integral en la Corporación Educativa Centro Superior de Cali. Tesis para obtener el grado de Magíster en Dirección Universitaria. Universidad de los Andes, Colombia; 2003.

11. Kaplan R, Norton D. El cuadro de mando integral. Barcelona: Ediciones Gestión 2000; 1997.

12. Rezzonico R, Miropolsky A. Uso del cuadro de mando integral en la evaluación de las instituciones de educación superior. Visitado 2007 May 15. Disponible en: http://www.posgrados.frc.unt.edu.ar

13. Bachelar G. La formación del espíritu científico. México: Editorial Siglo XXI; 1948.

14. Kim DA. Framework and methodology for linking Individual and Organization learning. Application in TQM and Product development. Cambridge, Mass: MIT Sloan School of Management; 1993.

15. Knowles M. Andragogy in action. San Francisco: Jossey-Bass; 1984. 